\title{
Design and analysis of a 3-DOF planar micromanipulation stage with large rotational displacement for micromanipulation system
}

\author{
Bingxiao Ding ${ }^{1}$, Yangmin $\mathbf{L i}^{1,2,3}$, Xiao Xiao ${ }^{1}$, Yirui Tang ${ }^{1}$, and Bin $\mathbf{L i}^{3}$ \\ ${ }^{1}$ Department of Electromechanical Engineering, University of Macau, Taipa, Macao SAR, China \\ ${ }^{2}$ Department of Industrial and Systems Engineering, The Hong Kong Polytechnic University, Hung Hom, Hong \\ Kong SAR, China \\ ${ }^{3}$ Tianjin Key Laboratory for Advanced Mechatronic System Design and Intelligent Control, Tianjin University \\ of Technology, Tianjin, China
}

Correspondence to: Yangmin Li (yangmin.li@ polyu.edu.hk)

Received: 28 October 2016 - Revised: 20 March 2017 - Accepted: 21 March 2017 - Published: 23 May 2017

\begin{abstract}
Flexure-based mechanisms have been widely used for scanning tunneling microscopy, nanoimprint lithography, fast servo tool system and micro/nano manipulation. In this paper, a novel planar micromanipulation stage with large rotational displacement is proposed. The designed monolithic manipulator has three degrees of freedom (DOF), i.e. two translations along the $X$ and $Y$ axes and one rotation around $Z$ axis. In order to get a large workspace, the lever mechanism is adopted to magnify the stroke of the piezoelectric actuators and also the leaf beam flexure is utilized due to its large rotational scope. Different from conventional pre-tightening mechanism, a modified pre-tightening mechanism, which is less harmful to the stacked actuators, is proposed in this paper. Taking the circular flexure hinges and leaf beam flexures hinges as revolute joints, the forward kinematics and inverse kinematics models of this stage are derived. The workspace of the micromanipulator is finally obtained, which is based on the derived kinematic models.
\end{abstract}

\section{Introduction}

Recently, flexure based mechanism with ultrahigh precision plays an increasing important role in many kinds of fields where high resolution and high repeatability accuracy are demanded, such as optical fiber alignment (Culpepper and Anderson, 2004), bioengineering ( $\mathrm{Li}$ and $\mathrm{Xu}, 2006$ ), scanning tunnel microscopy (STM) (Schitter et al., 2008). In precision applications such as following issues have attracted much attention: (1) resolution; (2) number of degrees of freedom(DOF); (3) workspace. The resolution refers to the capability of the system that can distinguish and detect the smallest change of the variable, in micromanipulation system this variable refers to the displacement of the end-effector other than the actuator, because of the amplification mechanism will degrade the system resolution (Ku et al., 2000). For planar mechanism, the maximum number of the DOF is three, i.e. translations along $X / Y$ axis and rotation around $Z$ axis, it can meet most of planar applications. The workspace of a manipulator is often defined as the set of points that can be reached by its end-effector, namely, it is the space in which the manipulator can work in either a 3-D space or a 2-D surface. The motion range of the micro manipulation stage is typically within several microns, for the reason of limited rotational scope of flexure hinges and limited stroke of piezoelectric actuators which are widely utilized in all kinds of flexure-based stages (Polit and Dong, 2011). For the purpose of getting better performance of the micromanipulation stage, more and more optimized flexure hinges with outstanding characteristics, such as more accurate in position and large motion scope, are designed by researchers (Acer and Sabanovic, 2013; Yi et al., 2003; Qin et al., 2013; Hao and $\mathrm{Li}, 2014$ ).

The stage should meet the design requirements when it is used in this specific application, this paper proposes a compliant parallel manipulator which adopts lever amplification 
mechanism to magnify the displacement of the piezoelectric actuators. Based on the structure of the flexure-based stage and the distribution of the actuators, manipulators can also be classified into two categories, i.e. serial structure and parallel structure. The serial structure of the stage enables a simple control strategy, however it endows with high inertia, low natural frequency and cumulative errors. To overcome such drawbacks, parallel kinematic structure is designed because it can provide high load capacity, low inertia, high accuracy and high stiffness. However, the disadvantages are limited workspace and complicated control strategies. Although the compliant parallel mechanism possesses such drawbacks, it has attracted much attention and become an hot issue.

During the literature review, the current research about flexure-based micromanipulation stages for planar application can be classified into 1-DOF, 2-DOF and 3-DOF manipulation stage. Although 1-DOF micromanipulator possesses the advantages of easy to control and no parasitic motion, it has limited applications. So the 2-DOF parallel symmetric micromanipulation stages with decoupled motion are proposed for many planar applications. However, to fully describe the movement of an object needs two translations and one rotation in planar applications, this paper presents a 3-DOF flexure-based parallel micromanipulation stage with large rotational scope for micromanipulation system. In micromanipulation application field, the operator often needs to adjust the position and orientation of biological objects under microscope to do cutting and filtering operation. As shown in Fig. 1, the stage is placed under the microscope, the operator can adjust the position of the object with the 3-DOF micro stage, it can make the manipulation more dexterous by operators.

During the literature review, a myriad of 3-DOF flexurebased compliant parallel mechanism has been fabricated by previous researchers. Generally speaking, the flexure-based micromanipulation stage has limited workspace, to overcome this drawback, many different kinds of amplification mechanisms are adopted to amplify the stroke of piezoelectric actuators. Lu et al. designed compliant parallel micro motion stage with two translations (along $X$ and $Y$ direction) and one rotation (around $Z$ axis), also adopted piezoelectric actuators, but without amplification mechanism, the drawback of this design is the very small workspace ( $\mathrm{Lu}$ et al., 2004); Bhagat et al. also designed a planar 3-DOF micromanipulator, this mechanism adopted three piezoelectric actuators to achieve required displacements in $X, Y$ and $\theta$ and utilized lever amplifier to enhance the displacement of the mechanism (Bhagat et al., 2014); Hao fabricated a flexure-based spatial 3-DOF compliant parallel mechanism with three translational motions along $X, Y$ and $Z$ axis, respectively, moreover this monolithic CPM can be used as positioning stage, acceleration sensor and energy harvesting device (Hao, 2013); Wang and Zhang proposed a 3DOF nanopositioning stage with two-level lever amplifier with over two hundreds of microns translational displace- ment along $x / y$, however the rotational scope and natural frequency are relatively small in Wang and Zhang (2016). In addition, other researchers have also designed many kinds of 3-DOF compliant parallel stages with different characteristics (Dong et al., 2016; Tian and Shirinzadeh, 2009).

The proposed flexure-based positioning stage is featured with two kinds of flexure hinges, right circular hinge and leaf beam flexure hinge in different places and an optimized lever amplification mechanism is adopted to compensate the limited stroke of piezoelectric actuators. The remainder of this paper is organized as follows: The flexure-based parallel positioning stage with optimized structure is proposed in Sect. 2, and also the rotational stiffness is compared between right circular hinge and leaf spring beam flexure hinge; In Sect. 3, the flexure-based mechanism is modeled based on PRB method and the pivot drifting analysis is also conducted; The kinematic analysis of this manipulation stage and the numerical simulation is conducted in Sects. 4 and 5 respectively; The dynamic characteristics and performance of this stage are evaluated in Sect. 6; Finally, the whole works of this research are concluded in Sect. 7 with future works indicated.

\section{Three-DOF micromanipulation-stage design}

For a planar 3-DOF parallel kinematic micromanipulation stage, the end-effector can translate along $X / Y$ axis and rotate around $Z$ axis. During the literature review, there are many kinds of 3-DOF planar mechanism configurations (Gao et al., 2002). Several typical planar parallel 3-DOF stages are shown in Fig. 2. For the (a) mechanism structural configuration, each chain has only revolute joint; the mechanisms (b) and (c) have prismatic joints and revolute joints. In this study, the (a) structure configuration is adopted to design the micromanipulation stage due to its easy to design and fabricate. When designing a flexure based micromanipulation stage, the structure, flexure hinges and actuators are needed to take into consideration, particularly, the goal of this study is to design a micromanipulation stage with large workspace and high resolution. Compared with shape memory alloy (SMA) and giant magnetostrictive actuator (GMA), the piezoelectric actuator is much cheaper and easily obtained, moreover the PEAs can achieve sub-nano level resolution and has fast response characteristics (Hubbard et al., 2006). Although the voice coil motor(VCM) and electromagnetic actuator (EMA) possess the advantages of large output stroke and driven force, the disadvantages are low resolution and slowly response. Considering all aspects, this design adopts PEA to drive the stage integrated with amplification mechanism to amplify the stroke of the PEAs.

\subsection{Flexure hinges}

Flexure hinges are basically designing elements in the flexure-based micromanipulation stages, which have been 


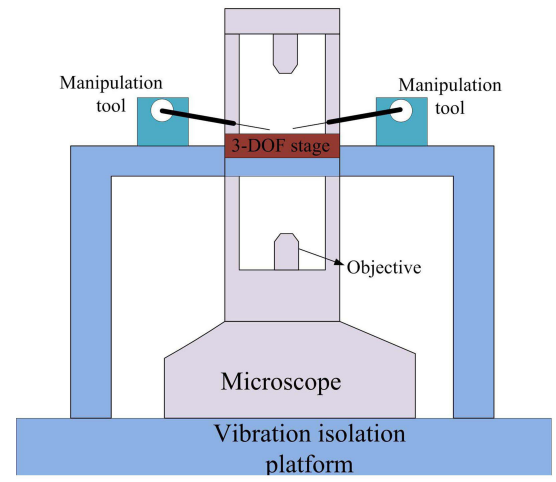

(a)

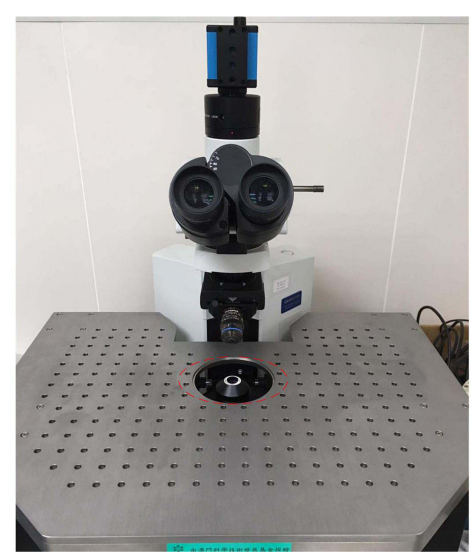

(b)

Figure 1. Micromanipulation system. (a) Schematic illustration. (b) Microscope picture.

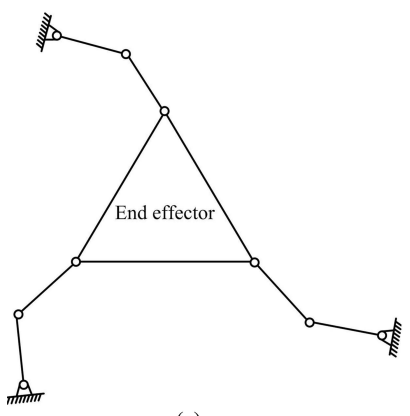

(a)

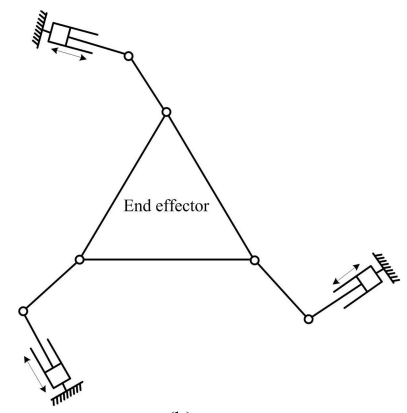

(b)

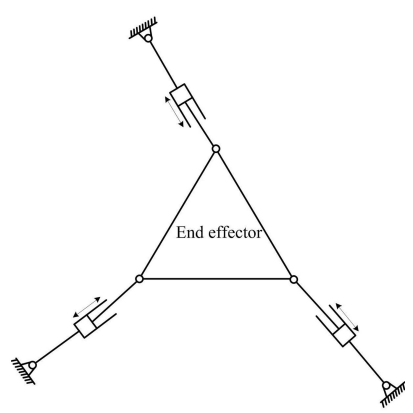

(c)

Figure 2. The typical planar parallel 3-DOF kinematic stages.

widely used in these applications where ultrahigh precision motion is needed such as aerospace field, high precision machine and bioengineering field. Furthermore, micromanipulation technology has become an important technology along with the appearance of using flexures. Compared with conventional joints, flexure hinges possess the advantages of no needing for lubrication, no hysteresis, no clearance and no wear. Therefore, flexure-based micromanipulation stages are capable of achieving highly precise positioning accuracy. The goal of this study is to design a compliant parallel micromanipulation stage with large workspace. Taking fabrication process and motion precision into consideration, this design chooses two types of flexures: leaf spring flexure and right circular cut flexure. The rotational stiffness of each type of flexure hinge can be calculated by the following equations, respectively (Smith, 2000):

$$
\begin{aligned}
K_{\theta_{z} M_{z}} & =\frac{E I}{2 a_{x}} \\
K_{\theta_{z} M_{z}} & =\frac{2 E b t^{5 / 2}}{9 \pi a_{x}^{1 / 2}}
\end{aligned}
$$

Here, $E$ represents the Young's modulus of aluminum alloy material, $I$ denotes the second moment of area about the neutral axis and $b$ is depth of the flexure hinge. What's more, the relationship between rotational stiffness of different notch types of flexure hinges and $t, a_{x}$ are illustrated in Fig. 3a, b respectively, here $E=71.7 \mathrm{GPa}, b=8 \mathrm{~mm}$. As Fig. 3 depicted, the leaf spring flexure has lower stiffness than right circular cut flexure with the same $t$ and $a_{x}$, therefore the leaf spring flexure hinge has a larger rotation than right circular cut flexure with the same driven force.

\subsection{Lever amplification mechanism}

The lever amplification mechanism is adopted to compensate the stoke of piezoelectric actuators for its advantage of simple mechanism and easy fabrication comparing with Scott-Russell (SR) Mechanism and Bridge Type Mechanism (BTM). As shown in Fig. 4, three types of lever amplification with different geometric forms have the same amplification ratio and the black dot line denotes the centroid of lever amplification mechanism. Moreover, the geometric form of the lever amplification mechanism will affect the acceleration of the end-effector. The inertia moment of each amplifier mechanism can be calculated by the following equation:

$$
J_{z}=\sum m_{i} r_{i}^{2}
$$




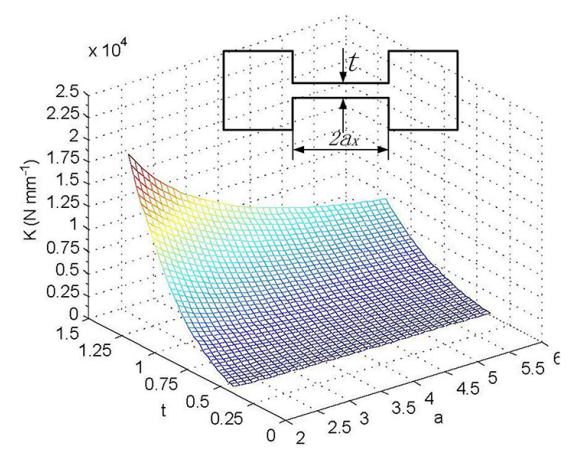

(a)

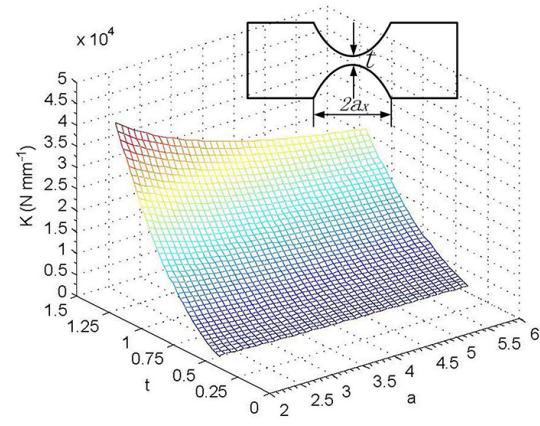

(b)

Figure 3. Different types of single DOF flexure hinge and its rotational stiffness with $t$ and $a_{x}$. (a) leaf spring flexure and its rotational stiffness; (b) right circular cut flexure and its rotational stiffness.
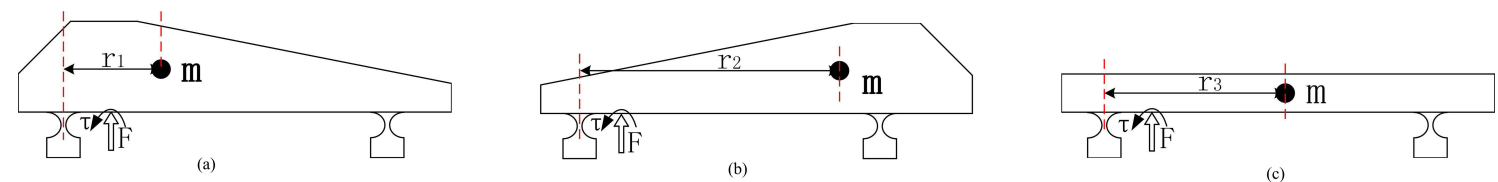

Figure 4. The lever amplification mechanism with different geometric forms.

where the $m_{i}$ denotes mass distribution and $r_{i}$ denotes the distance between each mass and rotational axis. So the following equation can be derived:

$$
\left\{\begin{array}{l}
J_{1}=m r_{1}^{2} \\
J_{2}=m r_{2}^{2} \\
J_{3}=m r_{3}^{2}
\end{array}\right.
$$

For the reason of $r_{1}<r_{3}<r_{2}$, the following relationship can be obtained:

$J_{1}<J_{3}<J_{2}$

With the same driving force $F$, inducing the same moment $\tau$, the acceleration of the end-effector with different geometries can be written as:

$\alpha_{i}=\frac{\tau}{J_{i}}$

where $i=1,2,3$. From above analysis, inducing $\alpha_{1}>\alpha_{3}>$ $\alpha_{2}$, it means that Fig. $4 \mathrm{a}$ is the reasonable geometry of lever amplification mechanism, (b) and (c) will degrade the response time of the manipulation stage.

\subsection{Preload mechanism}

It is well known that the piezoelectric actuator possesses the advantages of high resolution and fast response, however, it can not bear the lateral force since the lateral force or moment may cause the damage to the piezoelectric actuators, so shear stress and tensile stress must be avoided during the actuation. Also during the positioning process, the piezoelectric actuator needs to maintain a continuous connection state with the mobile platform. During the literature review, the typical pre-tightening mechanism uses the bolt to generate the thrust force and the bolt contact with piezoelectric directly, as shown in Fig. 5a, however the lateral force and moment can not be avoided and pre-load force can not be measured. A new pre-tightening mechanism is proposed in this study, as shown in Fig. 5b, the piezoelectric actuator and mobile platform maintain a line-face contact. The interaction of the semi-cylinder and the adjustable pre-load force block preclude the generation of the lateral force and bending moment.

\subsection{Structural configuration}

For a 3-RRR (R refers to revolute joint) parallel mechanism, it has two interesting configurations of the structure. As depicted in Fig. 6a and b, the same symbol denotes the same meaning, it means that the two schematic diagrams have the same geometric architecture configuration. The 3-RRR diagram (a) and (b) represent the structure with minimal rotation and maximal rotation, respectively. With the equal actuation force, the torque can be calculated by following equation:

$$
\begin{aligned}
& M_{1}=F_{1} \cdot\left(D B_{1}+C_{1} O\right)+F_{2} \cdot\left(D B_{2}+C_{2} O\right)+F_{3} \\
& \quad \cdot\left(D B_{3}+C_{3} O\right) \\
& M_{2}=F_{1} \cdot D B_{1}+F_{2} \cdot D B_{2}+F_{3} \cdot D B_{3}
\end{aligned}
$$

Obviously, $M_{1}>M_{2}$, it means that with the equal driving force, the (b) will generate a large rotational angle. So the 


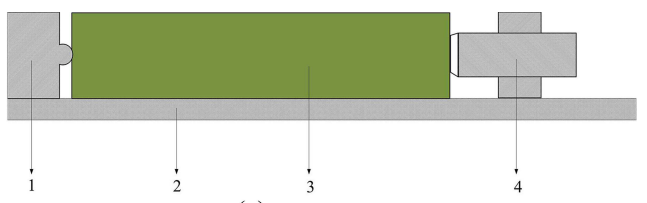

(a)

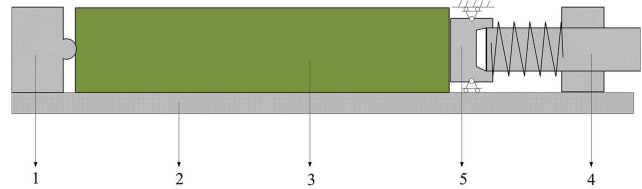

(b)

Figure 5. Pre-tightening mechanism. (a) traditional pre-tightening mechanism; (b) proposed pre-tightening mechanism; 1, mobile platform; 2, fixed platform; 3, piezoelectric actuator; 4, bolt; 5, adjustable pre-load force block.

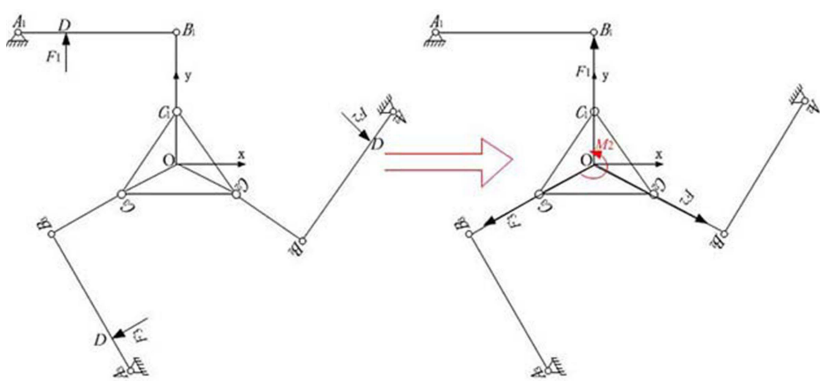

(a)

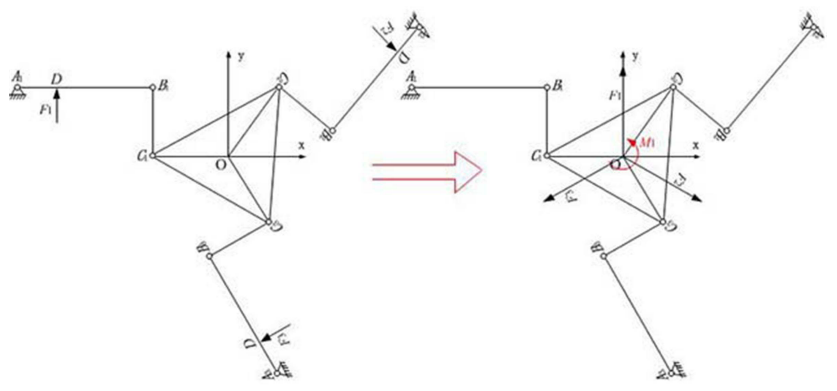

(b)

Figure 6. Two different 3-RRR structural configurations.

structural configuration (b) is adopted to design the 3-DOF micromanipulation stage for a large rotational scope.

Based on the above analysis, the designed monolithic 3DOF compliant parallel mechanism integrated with lever amplification mechanism is presented in Fig. 7. The proposed compliant parallel mechanism is designed to be used as a high precision planar positioning stage to manipulate objects under the microscopy and the dimension scale of this stage is approximately $120 \times 120 \times 8 \mathrm{~mm}^{3}$. In order to validate the correction of the designed mechanism, the Workbench software is adopted to simulate this parallel mechanism. As shown in Fig. 8, it does not exist any physical interferences under the condition of maximum input displacement, which indicates that the structural design is reasonable. The main design criteria of this compliant mechanism is to get a large workspace of the end-effector, to achieve this goal the beam flexures are exploited to connect the motion platform and lever amplification mechanism, meanwhile right circular hinges are adopted as revolute joints. The ex-

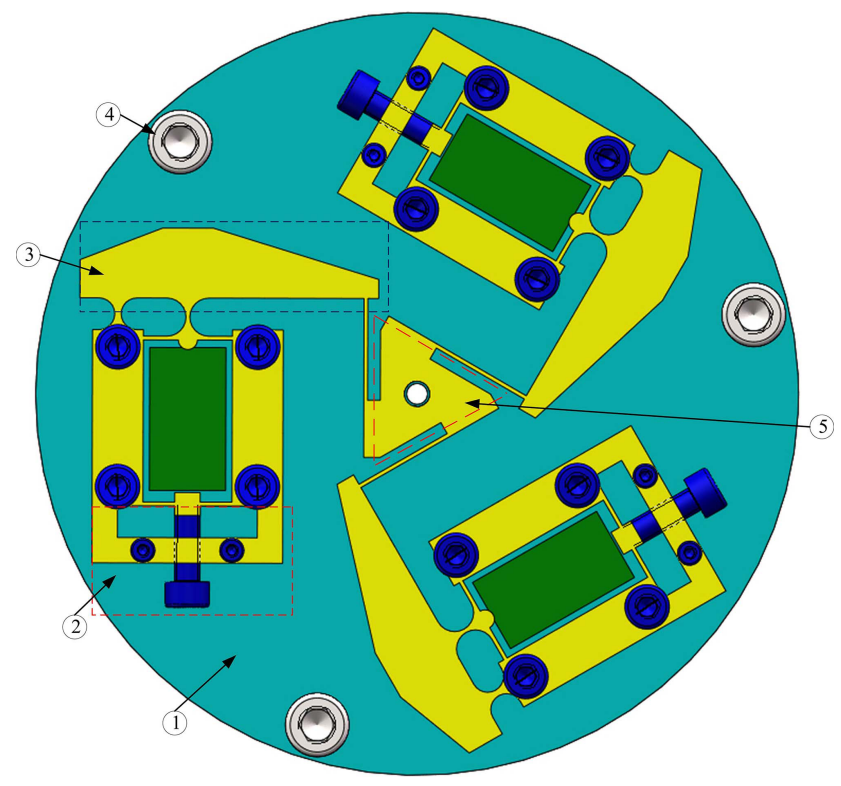

Figure 7. The whole assembled diagram of this three-DOF microstage: (1) The base platform; (2) The pre-tightening mechanism; (3) The lever amplifier; (4) The grounding bolt; (5) The central motion platform.

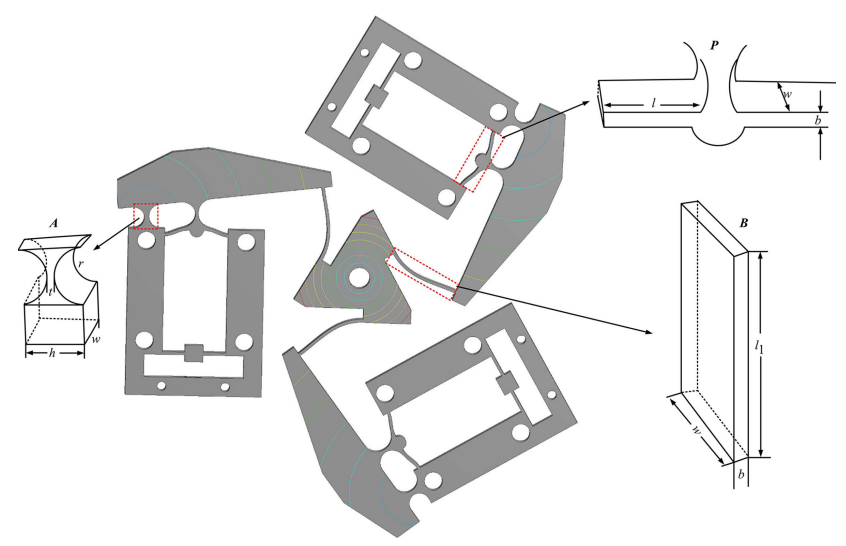

Figure 8. The motion simulation of manipulation stage with the condition of maximum input displacement.

act architectural parameters of flexure hinges are presented in Table 1. 
Table 1. Architectural parameters of the flexure hinges.

\begin{tabular}{lccccccc}
\hline parameter & value $(\mathrm{mm})$ & parameter & value $(\mathrm{mm})$ & parameter & value $(\mathrm{mm})$ & parameter & value $(\mathrm{mm})$ \\
\hline$t$ & 0.8 & $h$ & 8 & $l$ & 8 & $l_{1}$ & 16 \\
$r$ & 2.5 & $w$ & 8 & $b$ & 0.6 & & \\
\hline
\end{tabular}

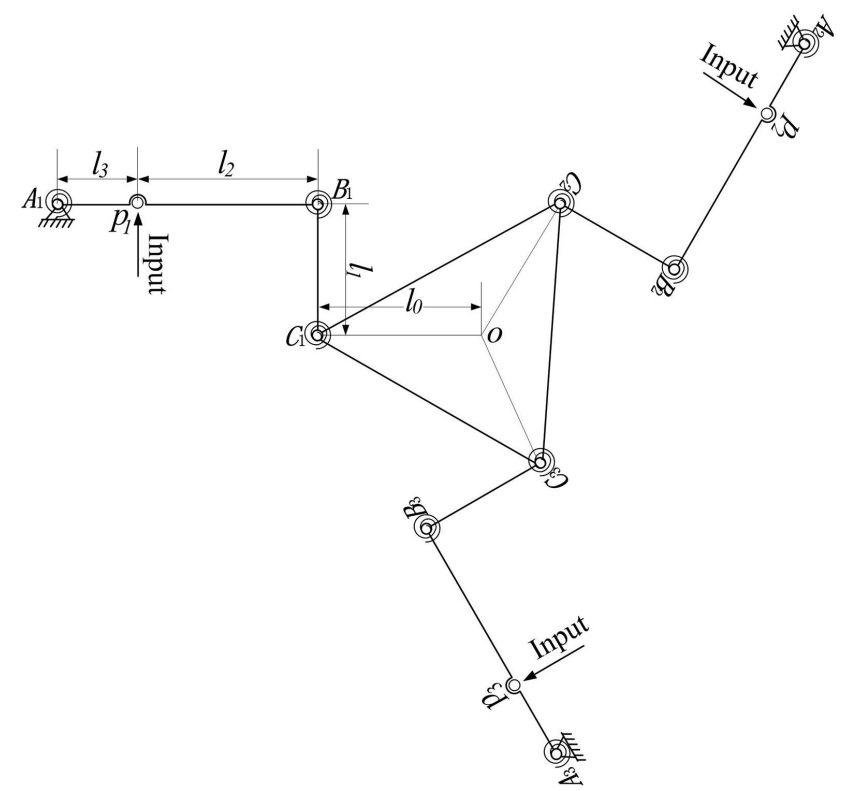

Figure 9. The PRB model of the designed compliant parallel stage.

\section{The micromanipulation stage modeling}

Compliant mechanisms involving with nonlinearity characteristics make the analysis and modeling very difficult. To facilitate the analysis of the manipulation stage, the pseudorigid-body (PRB) model is adopted in this study. With the identified mechanism topology and each flexure hinge replaced by a revolute joint and a torsional spring, the PRB model of the proposed flexure-based mechanism is developed as shown in Fig. 9. The stage is actuated by three stacked PZT actuators, where $P_{1}, P_{2}, P_{3}$ represents each actuator location and input driving force direction. The mobile platform $C_{1} C_{2} C_{3}$ is an equilateral triangle, which is connected by three identical linkages, namely, $A_{1} B_{1}=A_{2} B_{2}=$ $A_{3} B_{3}, B_{1} C_{1}=B_{2} C_{2}=B_{3} C_{3}$. The global frame $X O Y$ is grounded at the center of triangle $A_{1} A_{2} A_{3}$ and the local frame is attached at the center of platform $C_{1} C_{2} C_{3}$.

\subsection{Pivot drifting analysis}

The workspace of the manipulation stage depends on the actual input displacement of the piezoelectric actuator and the actual amplification ratio of the lever mechanism, not the theoretical amplification ratio. From the experience, the lever mechanism will produce bending deformation and pivot

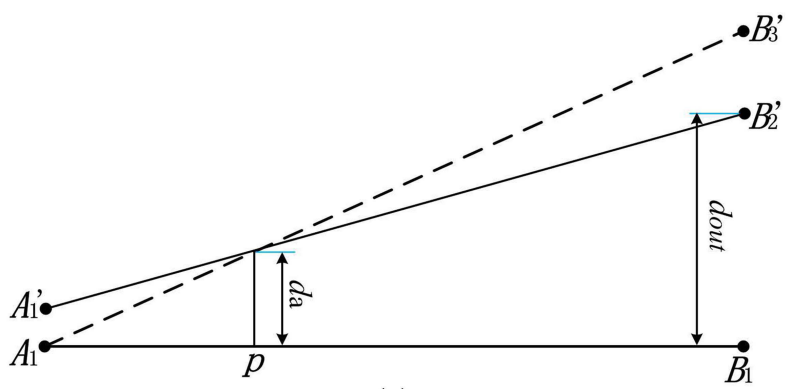

(a)

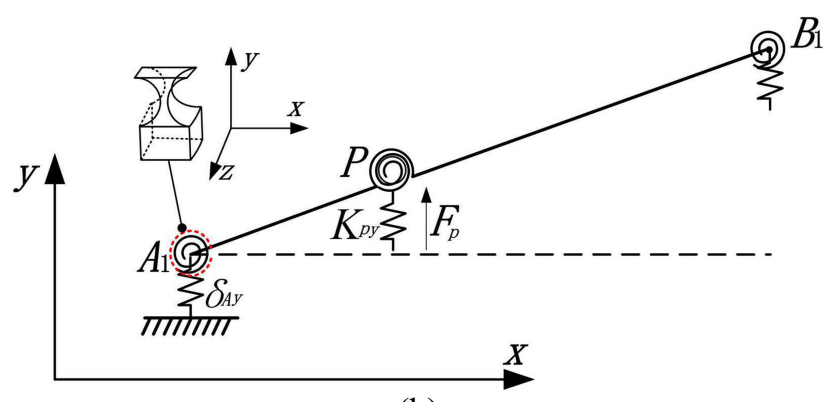

(b)

Figure 10. Lever amplifier mechanism. (a) Bending schematic diagram; (b) analysis model.

drifting when a loading is applied, the bending deformation can be ignored in this research for the geometric form of the lever amplifier mechanism. As shown in Fig. 10a, the theoretical magnification ratio of the mechanism is $A_{1} B_{1} / A_{1} p$, and the output displacement is $B_{1} B_{3}{ }^{\prime}$. However, the actual output displacement of the lever amplifier mechanism is $B_{1} B_{2}{ }^{\prime}$, when considering the displacement loss caused by the pivot drifting. Obviously, the actual amplification ratio $A_{\text {amp }}=d_{\text {out }} / d_{\mathrm{a}}$ is smaller than the theoretical ratio due to the displacement loss of the lever mechanism. The displacement loss needs to be taken into consideration in order to get a more accurate relationship between the input stroke and output displacement of the mobile platform.

As shown in Fig. 10b, when the driving force $F_{p}$ is applied on the mechanism, a pivot drift displacement $\delta_{A y}$ will be produced along the $y$ direction of the circular flexure hinge for its compliance in $y$ axis. And $\delta_{A y}$ can be solved by the following equation:

$\delta_{A y}=\frac{F_{p}}{K_{A y}}$ 


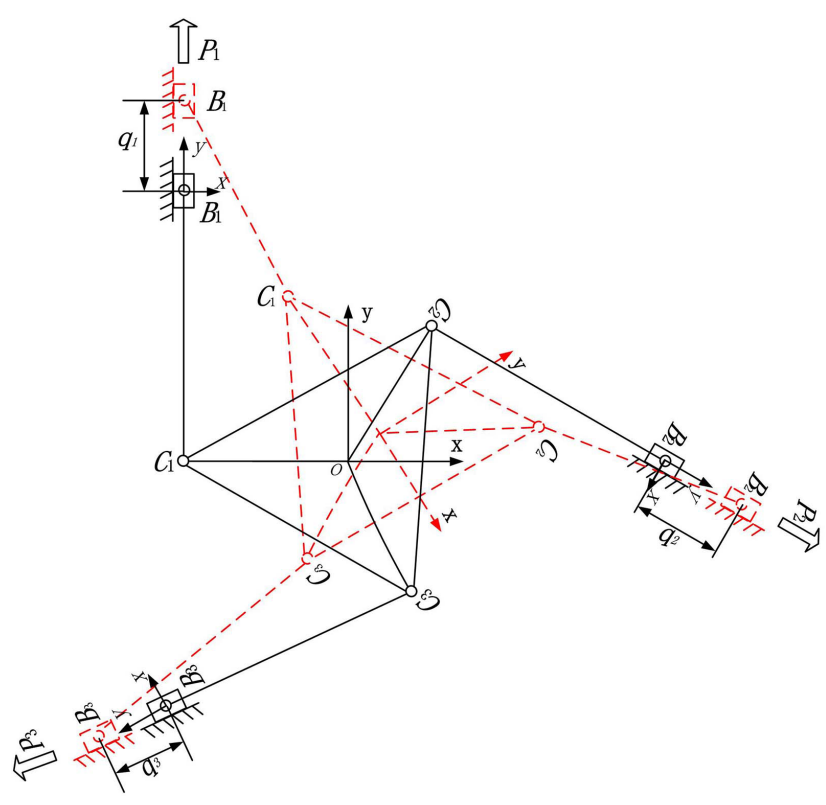

Figure 11. The kinematic schematic of 3-PRR planar mechanism.

where $K_{A y}$ is the stiffness of the circular hinge along the $Y$ axis, and it can be calculated by the following equation: Wu and Zhou (2002):

$K_{A y}=E w /\left\{\frac{2(2 r / t+1)}{\sqrt{4 r / t+1}} \arctan \sqrt{4 r / t+1}-\frac{\pi}{2}\right\}$

$F_{p}=K_{p y} \cdot d_{\mathrm{a}}$

where $K_{p y}=K_{p c}+K_{p b}$ denotes the total stiffness of the position $p$ along the driven direction, $K_{p c}=K_{A y}$ is the stiffness of the circular hinge along the $y$ axis and $K_{p b}=\frac{E b^{3} w}{4 l^{3}}$ represents the stiffness of the beam flexure hinge; $d_{\mathrm{a}}$ is the actual output displacement of the piezoelectric actuator; and the output displacement is $d_{\text {out }}=\left(d_{\mathrm{a}}-\delta_{A y}\right)\left(1+\frac{l_{2}}{l_{3}}\right)+\delta_{A y}$. So the relationship between the input displacement and the output displacement of the lever mechanism $A_{\text {amp }}$ can be derived as follows:

$\frac{d_{\text {out }}}{d_{\mathrm{a}}}=1+\frac{l_{2}}{l_{3}} \cdot \frac{b^{3} \cdot\left\{\frac{2(2 r / t+1)}{\sqrt{4 r / t+1}} \arctan \sqrt{4 r / t+1}-\frac{\pi}{2}\right\}}{4 l^{3}}$

Here, $E$ denotes the Young's modulus of the aluminum alloy material, and the parameters $w, t, r$ and $l$ are illustrated in Fig. 8. The amplification ratio is 3.2780 when taking the pivot drift into consideration, it exists about $8.16 \%$ deviation compared with nominal amplification ratio (3.5455); and exists about $3.68 \%$ deviation error compared with simulation result (3.1573).

\section{Kinematic analysis}

The kinematic diagram of the designed mechanism is presented in Fig. 11, $q_{i},(i=1,2,3)$ denotes the output dis- placement of the lever mechanism, it means that $q_{i}=d_{\text {out }}$. The workspace of mobile platform is defined by a vector $\boldsymbol{\mu}=(x, y, \theta)^{T}$, which represents the position and orientation of the reference point. And the input stroke of each stacked actuator can be denoted by $\boldsymbol{d}_{i},(i=1,2,3)$; the Jacobian matrix which connects the input stroke and output displacement of end-effector is denoted by $J$, so we can get the following equation:

$\left[\begin{array}{l}x \\ y \\ \theta\end{array}\right]=J\left[\begin{array}{l}d_{1} \\ d_{2} \\ d_{3}\end{array}\right]$

Here, we use $l_{0}, l_{1}$ to denote the length of $C_{i} O$ and $B_{i} C_{i}$, $(i=1,2,3)$, respectively, homogeneous transformation matrix from the global frame $X O Y$ to the coordinates $X O_{i} Y$ can be described by this equation:

${ }^{o} T_{i}=\left[\begin{array}{ccc}c_{i} & -s_{i} & l_{0} \\ s_{i} & c_{i} & -l_{1} \\ 0 & 0 & 1\end{array}\right]$

where $c_{i}=\cos \alpha_{i}, s_{i}=\sin \alpha_{i}, \alpha_{1}=0, \alpha_{2}=\frac{2 \pi}{3}, \alpha_{3}=\frac{4 \pi}{3}, i=$ $1,2,3$.

Also, the motion of the mobile platform can be described in the global frame $X O Y$.

${ }^{o} T_{O}=\left[\begin{array}{ccc}c & -s & x \\ s & c & y \\ 0 & 0 & 1\end{array}\right]$

where, $c=\cos \theta, s=\sin \theta$. Here, we adopt ${ }^{o} T_{i}$ to represent the homogeneous transformation matrix from the mobile platform to each coordinate $X O_{i} Y$. Combining the Eqs. (13) and (14) together, we can get the following equation:

${ }^{o} T_{i}=\left[\begin{array}{ccc}c_{i} & -s_{i} & l_{0} \\ s_{i} & c_{i} & -l_{1} \\ 0 & 0 & 1\end{array}\right]\left[\begin{array}{ccc}c & -s & x \\ s & c & y \\ 0 & 0 & 1\end{array}\right]$

Here, we define the $s=\theta, c=1$, because the rotational angle of the platform is very tiny. After a further processing of Eq. (15), we can obtain the relatively simple ${ }^{o} T_{i}$ as follows:

${ }^{o} T_{i}=\left[\begin{array}{ccc}c_{i}-s_{i} \theta & -c_{i} \theta-s_{i} & x c_{i}-y s_{i}+l_{0} \\ s_{i}+C_{i} \theta & c_{i}-s_{i} \theta & x s_{i}+y c_{i}-l_{1} \\ 0 & 0 & 1\end{array}\right]$

In the frame of $X O Y$, the coordinates value of $C_{i}$, $(i=1,2,3)$ can be expressed as ${ }^{o} C_{1}=\left(-l_{0}, 0\right),{ }^{o} C_{2}=$ $\left(\frac{l_{0}}{2}, \frac{\sqrt{3} l_{0}}{2}\right),{ }^{o} C_{3}=\left(\frac{l_{0}}{2}, \frac{-\sqrt{3} l_{0}}{2}\right)$.

$\left\{\begin{array}{l}{ }^{i} C_{i} x=\left(c_{i}-s_{i} \theta\right)^{o} C_{i} x-\left(c_{i} \theta+s\right)^{o} C_{i} y+x c_{i}-y s_{i}+l_{0} \\ { }^{i} C_{i} y=\left(s_{i}+c_{i} \theta\right)^{o} C_{i} x-\left(s_{i} \theta c s_{i}\right)^{o} C_{i} y+x s_{i}-y c_{i}-l_{1}\end{array}\right.$ 
where $i=1,2,3$. For the length of $B_{i} C_{i}$ is constant constraint, we can obtain the following equation:

$\left(q_{i}+{ }^{i} C_{i} y\right)^{2}+{ }^{i} C_{i} x^{2}=l_{1}^{2}$

So the $q_{i}$ can be calculated by the following equation:

$q_{i}=l_{1} \sqrt{1-\left(\frac{{ }^{i} x}{l_{1}}\right)^{2}}-{ }^{i} C_{i} y$

The above Eq. (19) is a nonlinear equation, after further analysis, we can get ${ }^{i} C_{i} x / l_{1} \approx 0$, subsequently, we can get the following linearized equation:

$q_{i}=l_{1}-{ }^{i} C_{i} y$

We can obtain the following equations after substituting (17) into (20).

$$
\left\{\begin{array}{l}
q_{1}=l_{0} \theta+y \\
q_{2}=l_{0} \theta-\frac{\sqrt{3}}{2} x-\frac{1}{2} y \\
q_{3}=l_{0} \theta+\frac{\sqrt{3}}{2} x-\frac{1}{2} y
\end{array}\right.
$$

So the relationship between input stroke of piezoelectric actuators and the position and orientation of the central platform can be written as follows:

$$
\left[\begin{array}{l}
x \\
y \\
\theta
\end{array}\right]=A_{\text {amp }}\left[\begin{array}{ccc}
0 & -\frac{\sqrt{3}}{3} & -\frac{\sqrt{3}}{3} \\
\frac{2}{3} & -\frac{1}{3} & -\frac{1}{3} \\
\frac{1}{3 l_{0}} & \frac{1}{3 l_{0}} & \frac{1}{3 l_{0}}
\end{array}\right]\left[\begin{array}{l}
d_{1} \\
d_{2} \\
d_{3}
\end{array}\right]
$$

where the $A_{\text {amp }}$ is the actual amplification ratio of the lever amplifier.

\section{Workspace analysis}

The workspace of the 3-DOF manipulator can be calculated by the above aforementioned kinematic analysis. Actually, the reachable workspace of the manipulator not only depends on the above Eq. (22), but also has the relationship with material property. The following list is the constraint condition of the 3-DOF micromanipulator:

$$
\left\{\begin{array}{l}
0 \leq d_{i} \leq d_{\max }, \quad i=1,2,3 \\
\sigma<\sigma_{m}
\end{array}\right.
$$

where $d_{i}$ denotes the output displacement of the piezoelectric actuators; and $\sigma$ is the stress of the material.

Here, the piezoelectric actuators $P-820.20$ are adopted to drive the stage, so the first constraint condition is the input stroke of each piezoelectric actuator:

$0 \leq d_{i} \leq 20$

where $i=1,2,3$.
Table 2. Length parameters of microstage.

\begin{tabular}{lc}
\hline parameters & values $(\mathrm{mm})$ \\
\hline$A_{1} B_{1}$ & 39 \\
$B_{1} C_{1}$ & 16 \\
$C_{1} C_{2}$ & 12 \\
$A_{1} P_{1}$ & 11 \\
\hline
\end{tabular}

Based on above analysis, the workspace of the micromanipulator can be obtained by calculating the outputs of the reference point. The 3-D workspace is graphically illustrated in Fig. 12a. From the simulation results, the reachable workspace of the end-effector is a hexahedron, and the projection area on $X Y$ plane is varying with the $\theta$ value. The maximum projection on the $X Y$ plane is shown in Fig. 12b. From the simulation results, the reachable workspace along $X / Y$ axis and around $Z$ is $[-42.31,42.31] \mu \mathrm{m},[-48.56,48.56] \mu \mathrm{m},[0,10.28] \mathrm{mrad}$, respectively.

\section{Dynamic characteristics}

The dynamic response has to be modeled in order to fully describe the free vibrations of the 3-DOF micro manipulation stage and ensure the flexure-based mechanism operate properly in the dynamic range. Referring to the PRB method, the flexure hinges generated rotational motion around $Z$ axis are treated as an ideal revolute joint with torsional spring. The output variables of the end-effector are defined as $\overrightarrow{\boldsymbol{O}}=$ $\left[x_{o}, y_{o}, \theta_{o}\right]^{T}$. During the simulation analysis, the motion type of the lever $A_{i} B_{i}(i=1,2,3)$ is mainly rotational motion, and beam links $B_{i} C_{i}(i=1,2,3)$ will be generated bending deformation when the input is applied. So the total kinematic energy of the manipulation stage is composed by the translational kinematic energy $T_{t}$ of the end-effector and rotational kinematic energy $T_{r}$ of the lever $A_{i} B_{i}(i=1,2,3)$ and the end-effector.

$$
\begin{array}{r}
T_{r}=\frac{1}{2} \sum_{i=1}^{3} J_{A_{i} B_{i}} \omega_{A_{i} B_{i}}^{2}+\frac{1}{2} J_{o} \dot{\theta}_{o}^{2} \\
T_{t}=\frac{1}{2} m_{o}\left(\dot{x}_{o}^{2}+\dot{y}_{o}^{2}\right)
\end{array}
$$

where $J_{A_{i} B_{i}}$ denotes the moment of inertia of each lever; $\omega_{A_{i} B_{i}}$ represents the angular velocity of each lever. As illustrated in Fig. 8, the potential energy mainly concentrates on beam type flexure hinges at position $P_{i}(i=1,2,3)$ and beam links $B_{i} C_{i}(i=1,2,3)$ when input force is applied. So the total potential energy $V$ of the manipulation stage can be calculated by the following equation:

$$
V=\frac{1}{2} K_{x} x_{o}^{2}+\frac{1}{2} K_{y} y_{o}^{2}+\frac{1}{2} K_{\theta} \theta_{o}^{2}+\frac{3}{2} K_{p b}\left(\frac{d_{\text {out }}}{A_{\text {amp }}}\right)^{2}
$$




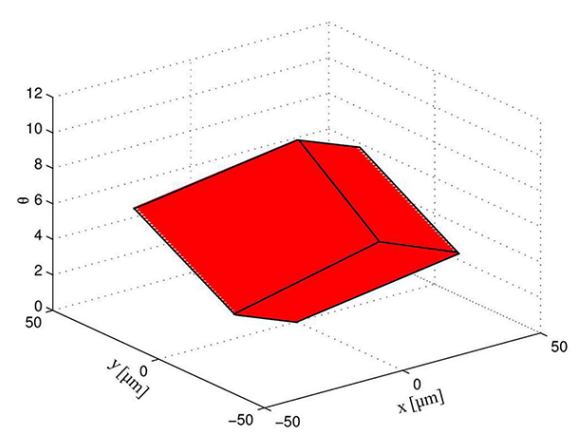

(a)

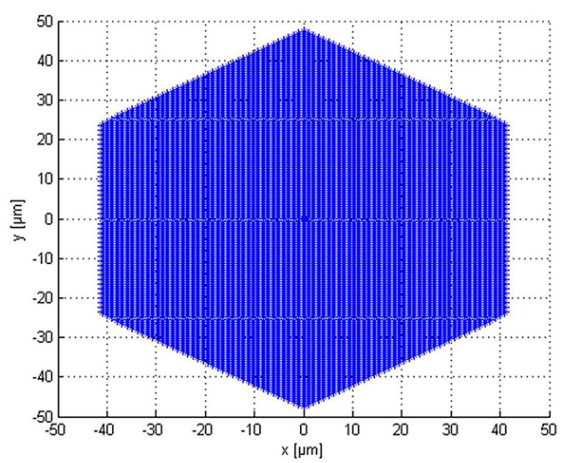

(b)

Figure 12. Reachable workspace of the stage. (a) 3-D workspace; (b) maximum projection on $X Y$ plane.

where the $K_{x}, K_{y}, K_{\theta}$ denotes the equivalent stiffness of the micro manipulation stage along $X / Y$ axis and around $Z$ axis, respectively. The numerical solution of $K_{x}, K_{y}$, and $K_{\theta}$ can be obtained by some experimental techniques. Substituting the potential energy and kinematic energy into the Lagrange's equation can yield to the following equation:

$M\left[\begin{array}{lll}\ddot{x}_{o} & \ddot{y}_{o} & \ddot{\theta}_{o}\end{array}\right]^{T}+K\left[\begin{array}{lll}x_{o} & y_{o} & \theta_{o}\end{array}\right]^{T}=\left[\begin{array}{lll}F_{1} & F_{2} & F_{3}\end{array}\right]^{T}$

where $\boldsymbol{F}_{i}(i=1,2,3)$ is the $i$ th input force generated by piezoelectric actuator. Then the following mass-stiffness matrix characteristic equation can be obtained:

$\left|K-M \lambda_{i}^{2}\right|=0$

So the natural frequencies can be calculated by $f_{i}=\lambda_{i} / 2 \pi$.

In this section, the dynamic characteristics of the manipulation stage are obtained through the modal analysis by Workbench. The first four modal shapes of the micromanipulation stage without piezoelectric actuator assembled are represented in Fig. 13. The first four natural frequencies are $1280.3,1308.4,1618.6$ and $1918.2 \mathrm{~Hz}$. It should be noticed that dynamic performance of micro-manipulation stage will be mainly determined by some experimental procedures, which are planned in our future work and the aforementioned analysis and simulation results will be validated.

\section{Conclusions}

A flexure-based monolithic micro manipulation stage with large workspace is designed and analyzed in this paper. The piezoelectric actuators are adopted to drive the manipulation stage because they can provide with the fast response and large output driven force. The optimized lever amplifier is integrated into the mechanism in order to compensate the stroke of the piezoelectric actuators. For the purpose of getting larger workspace, different kinds of flexure hinges, different structural configurations and lever amplifiers with different geometric forms are compared in this paper. To validate the design, the kinematics model and reach-

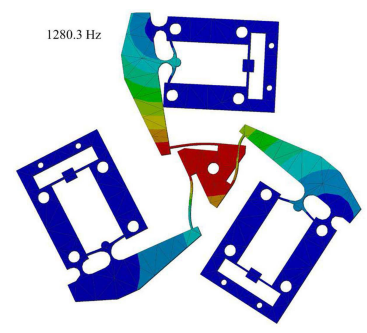

(a)

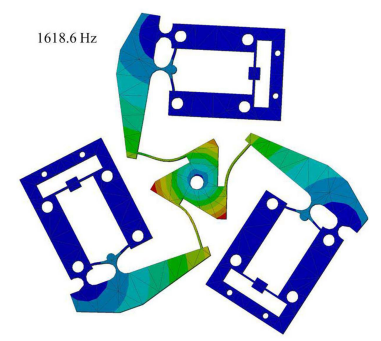

(c)

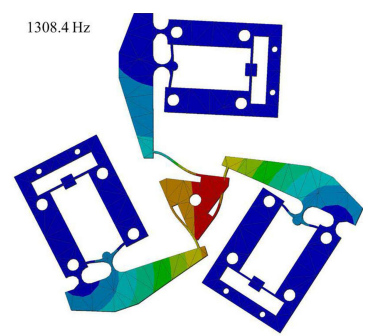

(b)

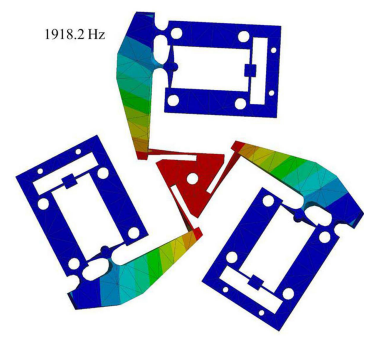

(d)
Figure 13. The lever amplification mechanism with different geometric forms.

able workspace of the manipulation stage are analytically obtained. The working range of the manipulation stage along each axis is $\pm 42.31 \mu \mathrm{m}, \pm 48.56 \mu \mathrm{m}, 0-10.28 \mathrm{mrad}$, respectively. Then the dynamic characteristics and performance evaluation of the designed mechanism are conducted via the Workbench software. The prototype fabrication and experimental validations, and control of the micro manipulation stage will be performed in our future work.

Data availability. All the data used in this manuscript can be obtained by requesting from the corresponding author. 
Competing interests. The authors declare that they have no conflict of interest.

Acknowledgements. This work is partially supported by National Natural Science Foundation of China (51575544,51275353), Macao Science and Technology Development Fund (110/2013/A3,108/2012/A3), Research Committee of University of Macau (MYRG2015-00194-FST, MYRG203(Y1-L4)-FST11LYM).

Edited by: G. Hao

Reviewed by: two anonymous referees

\section{References}

Acer, M. and Sabanovic, A.: Micro position control of a designed 3-PRR compliant mechanism using experimental models, 9th Asian Control Conference, 1-6, 2013.

Bhagat, U., Shirinzadeh, B., Clark, L., Chea, P., Qin, Y. D., Tian, Y. L., and Zhang, D. W.: Design and analysis of a novel flexurebased 3-DOF mechanism, Mech. Mach. Theory, 7, 173-187, 2014.

Culpepper, M. L. and Anderson, G.: Design of a low-cost nanomanipulator which utilizes a monolithic, spatial compliant mechanism, Precision Engineering, 28, 469-482, 2004.

Dong, Y., Gao, F., and Yue, Y.: Modeling and experimental study of a novel 3-RPR parallel micro-manipulator, Robotics and Computer-IntegratedManufacturing, 37, 115-124, 2016.

Gao, F., Li, W. M., Zhao, X. C., Jin, Z. L., and Zhao, H.: New kinematic structures for 2-, 3-, 4-, and 5-DOF parallel manipulator designs, Mech. Mach. Theory, 37, 1395-1411, 2002.

Hao, G.: Towards the design of monolithic decoupled XYZ compliant parallel mechanisms for multi-function applications, Mech. Sci., 4, 291-302, doi:10.5194/ms-4-291-2013, 2013.

Hao, G. B. and Li, H. Y.: Design of 3-legged XYZ compliant parallel manipulators with minimised parasitic rotations, Robotica, 33, 787-806, 2014.
Hubbard, N. B., Culpepper, M. L., and Howell, L. L.: Actuators for micropositioners and nanopositioners, Appl. Mech. Rev., 59, 324-334, 2006.

Ku, S. S., Pinsopon, U., Cetinkunt, S., and Nakajima, S. I.: Design, fabrication, and real-time neural network control of a threedegrees-of-freedom nanopositioer, IEEE/ASME Transactions on Mechatronics, 5, 273-280, 2000.

Li, Y. M. and Xu, Q. S.: A novel design and analysis of a 2DOF compliant parallel micromanipulator for nanomanipulation, IEEE Transactions on Automation Science and Engineering, 3, 248-254, 2006.

Lu, T. F., Handley, D. C., Yong, Y. K., and Eales, C.: A threeDOF compliant micromotion stage with flexure hinges, Industrial Robot: An International Journal, 31, 355-361, 2004.

Polit, S. and Dong, J.: Development of a high-bandwidth XY nanopositioning stage for high-rate micro-/nanomanufacturing, IEEE/ASME Transactions on Mechatronics, 16, 724-733, 2011.

Qin, Y. D., Shirinzadeh, B., Zhang, D. W., and Tian, Y. L.: Design and kinematics modeling of a novel 3-dof monolithic manipulator featuring improved Scott-Russell mechanisms, Journal of Mechanical Design, 135, 1-9, 2013.

Schitter, G., Thurner, P. J., and Hansma, P. K.: Design and inputshaping control of a novel scanner for high-speed atomic force microscopy, Mechatronics, 18, 282-288, 2008.

Smith, S. T.: Flexures: elements of elastic mechanisms, CRC Press, 2000.

Tian, Y. L. and Shirinzadeh, B.: Development of a flexure-based 3-RRR parallel mechanism for nano-manipulation, IEEE/ASME International Conference on Advanced Intelligent Mechatronics, 1324-1329, 2009.

Wang, R. Z. and Zhang, X. M.: A planar 3-DOF nanopositioning platform with large magnification, Precision Engineering, 46, 221-231, 2016.

Wu, Y. F. and Zhou, Z. Y.: Design of flexure hinges, Eng. Mech., 19, 136-140, 2002.

Yi, B., Chung, G., Na, H., Kim, W., and Suh, I.: Design and experiment of a 3-DOF parallel micromechanism utilizing flexure hinges, IEEE Transactions on Robotics and Automation, 19, 604-612, 2003. 